\title{
Desarrollo de la Actividad Científica en Clases a través del Estudio de Juegos Combinatorios, el Ejemplo del Juego del Chocolate
}

\author{
Development of Scientific Activity in the classroom through the study of \\ Combinatorial Games, the Example of the Chocolate Game
}

Ximena Colipan ${ }^{*}$

\begin{abstract}
Resumen
La matemática discreta es la fuente de numerosos juegos matemáticos. Su presencia en olimpiadas matemáticas, revistas de divulgación científica y festivales de ciencia nos muestra que esta rama puede ser un potencial soporte para un encuentro con la matemática accesible a un gran número de personas. El modelo Situations de recherche pour la classe ( $\mathrm{SiRC}$ ) es un modelo didáctico francés creado hace 20 años con el objetivo de trabajar los saber-hacer fundamentales de la actividad matemática. Estas situaciones permiten, sobre la base de conocimientos elementales, entrenar a los alumnos desde la educación básica hasta la educación superior a una práctica genuina de la matemática dándoles el rol de investigador. Este artículo muestra parte de los resultados de nuestras investigaciones sobre el estudio de juegos combinatorios (un objeto de estudio de la matemática discreta) y su trasposición didáctica en clases a través de las SiRC. Específicamente, mostraremos el análisis didáctico del juego del Chocolate y la experimentación realizada con estudiantes de primer año de la carrera de pedagogía en Matemática de una universidad Chilena.
\end{abstract}

Palabras-clave: Situations de recherche pour la classe (SiRC). Juegos combinatorios. Ingeniería didáctica.

\begin{abstract}
Discrete mathematics is the source of several mathematical games. Its presence in Mathematical Olympiads, popularization of science magazines, and science festivals shows that this area of mathematics may be the support for a first encounter with accessible mathematics for a great number of people. The model "Situations de recherche pour la classe" ( $\mathrm{SiRC}$ ) is a french didactic model created 20 years ago with the aim of working the fundamental know-hows of mathematical activity. These situations allow, in the base of elementary knowledge, to train students from primary to higher education on a genuine practice of mathematics giving them the role of the researcher. This paper shows part of the results from our research on the study of combinatorial games (part of discrete mathematics) and its didactic transposition to the classroom trough the SiRC model. Specifically, we show the didactical analysis of the Chocolate Game and the experimentation conducted on first year student of mathematics teaching in a Chilean University.
\end{abstract}

Keywords: Situations de recherche pour la classe (SiRC) . Combinatorial games. Didactic Engineering.

\footnotetext{
" Doctora en Didáctica de la matemática por la universidad Joseph Fourier (UJF), Grenoble I, Francia. Investigadora asociada de la Universidad Católica del Maule, Talca, Región del Maule, Chile. Dirección postal: Avda. San Miguel 3605, 3460000, Talca, Chile. E-mail: Ximena.colipan@gmail.com.
} 


\section{Problema y antecedents}

Una de las preocupaciones sociales relativa a las ciencias, es la gran desafección de los estudiantes hacia el área científica, que se complementa, además, con los bajos resultados que obtienen los alumnos a medida que avanzan es su escolarización.

Se ha apuntado como posible causa, de esta desmotivación, al tratamiento que se le da en clases a las disciplinas asociadas, es decir, Física, Química, Biología y Matemática. En efecto, tal como plantea (HALL, 2012) generalmente estas disciplinas son estudiadas como entes complejos, orientadas hacia el aprendizaje de nociones y procesos más abstractos que palpables. A lo anterior se suma la imposición de contenidos curriculares que dejan al alumno con poca o ninguna oportunidad de participar en la planificación de su propia formación.

De esta manera imperan los tradicionales métodos de enseñanza-aprendizaje, clases muy teóricas, poco participativas, además de inflexibles, que lejos de atraer y estimular, fastidian, desmotivan y extirpan la creatividad e interés por las ciencias (COLIPAN; GRENIER, 2014).

Estos factores que sesgan el interés hacia las ciencias nos lleva a reflexionar sobre otro elemento importante: la gran distancia que existe entre el contenido de las prácticas escolares y la realidad de la investigación científica, en efecto, erróneamente muchos piensan que el conocimiento científico se articula en forma de algoritmos y definiciones que deben ser memorizadas más que comprendidas (CAMPANARIO; MOYA; 1999; GRAVIER; OUVRIER-BUFFET, 2009). Lo anterior constituye un obstáculo enorme para el aprendizaje de las ciencias en general y puede ser uno de los responsables del fracasos a nivel motivacional (TISSERON, 1996; GODOT, 2005; OUVRIER-BUFFET, PERRIN, 2009). Entonces, nos parece necesario integrar a la clase situaciones que tengan como objetivo enseñar a los alumnos a probar, testear hipótesis, a razonar sobre problemas que no tienen necesariamente relación con los aprendizajes de la clase, que no tienen como objetivo participar en la construcción de alguna noción específica, si no que aprender a investigar y concluir positivamente esta búsqueda (COPPÉ; HOUDEMENT, 2002; GODOT, 2005; GIROUD, 2011; COLIPAN, GRENIER, 2014).

En este contexto, es que nosotros nos interesamos en la construcción de situaciones que por un lado sean próximas a la investigación y que por otro sean lúdicas y motivantes. Nuestro objetivo es recrear la actividad científica de un investigador en matemática, para construir y desarrollar los saber-hacer fundamentales de la actividad matemática en clases. 
Consideramos como saber-hacer fundamentales, los saberes, métodos y técnicas que son base de toda actividad matemática: la experimentación, el estudio de casos particulares, el enunciado y estudio de conjeturas, la construcción de ejemplos y contra-ejemplos, la modelización, la elaboración y escritura de pruebas, la definición de objetos etc. (GRENIER; PAYAN, 2002).

La modalidad consiste en resolver problemas próximos a la investigación. Las características de estos problemas están basadas en el modelo francés Situations Recherche pour la Classe (SiRC), (GRENIER, PAYAN, 1998; GODOT, 2005; GIROUD, 2011; COLIPAN, 2014) el cual presentamos en la sección 2. Estas situaciones permiten, sobre la base de conocimientos elementales, entrenar a los alumnos desde la educación básica hasta la educación superior a una práctica genuina de la matemática dándoles el rol de investigador.

La siguiente investigación muestra el estudio de la transposición didáctica de los juegos combinatorios, objeto de estudio de la matemática discreta, para el aprendizaje de los saber-hacer fundamentales de la actividad matemática. En especial, mostramos el ejemplo del juego del Chocolate.

\section{Marco teórico y metodología}

\section{1 ¿Qué es un juego combinatorio? Definiciones y terminología}

Los juegos han existido durante toda nuestra historia, pero la aplicación sistemática de las matemáticas hacia los juegos es un fenómeno relativamente reciente. La teoría de juegos combinatorios es una teoría matemática que estudia los juegos de dos jugadores, que juegan por turnos, con reglas definidas, con el objetivo de llegar a una cierta condición de victoria (BERLEKAMP; CONWAY; GUY, 2001).

Un famoso juego de esta teoría es el juego de Marienbad (del film El año pasado en Marienbad) del cual presentamos un ejemplo a continuación.

Regla del juego: El juego consta de dos jugadores y cuatro filas de objetos con uno, tres, cinco y siete elementos respectivamente. Los jugadores, deben, en cada uno de sus turnos retirar uno o más objetos de una sola fila (también los puede retirar todos). Pierde el juego quien retire el último objeto. 


\section{Figura 1 - Posición inicial del juego de Marienbad}

Ejemplo: En el siguiente esquema, vemos al primer jugador en azul a la derecha y el segundo jugador en rojo a la izquierda. En la primera jugada, azul toma una carta de la cuarta fila y rojo toma una carta de la tercera fila.
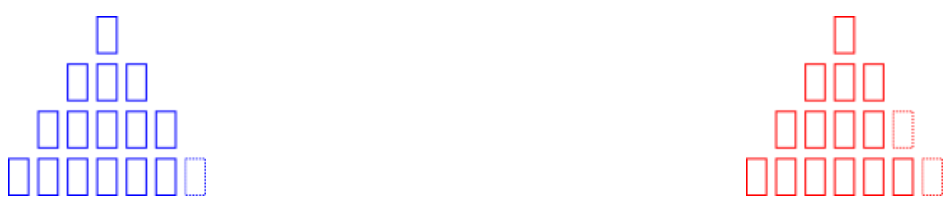

Figura 2 - Primer turno

Después, azul toma todo lo que queda de la cuarta fila y rojo toma dos cartas de la tercera fila.

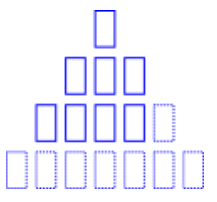

Figura 3 - Segundo turno

En el tercer turno, azul toma una carta de la tercera fila y rojo toma dos cartas de la segunda.
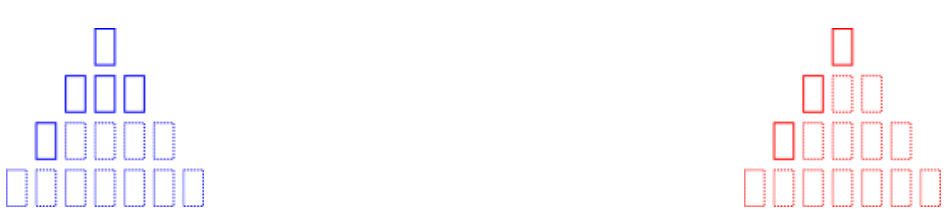

\section{Figura 4 - Tercer turno}

En el turno siguiente, azul toma la carta de la primera fila y rojo la carta que queda de la segunda fila.
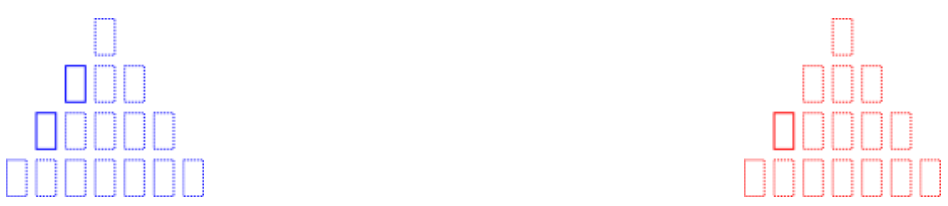

Figura 5 - Cuarto turno

Finalmente, azul toma la carta que queda, por lo que pierde la partida.

El juego de Marienbad es una variante de un juego mucho mas famoso llamado el Juego de Nim, que ha inspirado muchos trabajos en matemática (BOUTON, 1901). Uno de esto trabajos es el de Berlekamp, Conway y Guy (2001), que es el punto de partida de una teoria en los años 60, el conjunto de estos resultados fue publicado 1982, en su libro Winning 
Way four your Mathematical Plays, y hasta nuestros días es el libro de referencia de la teoria de juegos combinatorios.

\subsubsection{Caracteristicas de un juego combinatorio}

Berlekamp, Conway y Guy (2001) definieron, de forma precisa, lo que es un juego combinatorio, esta definición fue adoptada por la comunidad matemática del área y está compuesta de 8 axiomas (ver también DUCHENE, 2006):

1. El juego consta de 2 jugadores, que jugan alternadamente.

2. El juego esta determinado por un numero finito de posiciones. La posición de partida o inicial es conocida por ambos jugadores.

En el ejemplo anterior, el juego de Marienbad, la posición inicial es:

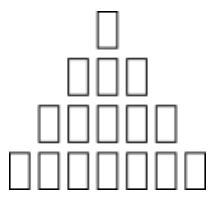

Figura 6 - Posición inicial del juego de Marienbad

3. La regla del juego precisa claramente las jugadas que se pueden realizar en cada turno a partir de una posición dada. Las posiciones son denotadas por P y el conjunto de posiciones accesibles en una sola jugada es llamada opciones de P, denotada por opt $(P)$.

Considerando estas definiciones, hacer una jugada a partir de una posición, corresponde a elegir una de las opciones de esta posición. Por ejemplo, para la siguiente posición del juego de Marienbad:

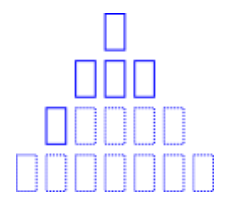

Figura 7 - Posición $P$ del ejemplo del juego de Marienbad

El conjunto de opciones es:
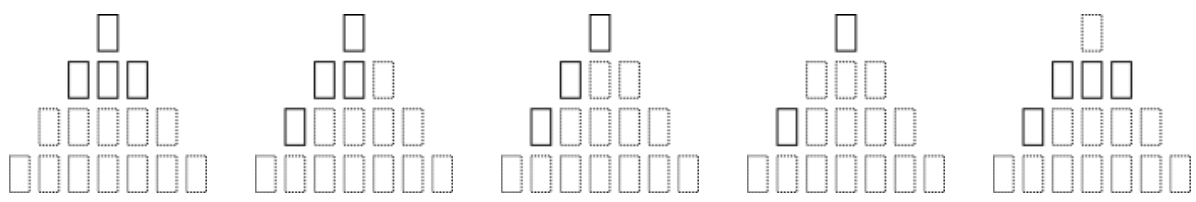

Figura 8 - conjunto de opt $(P)$, de la posición $P$

4. Los jugadores juegan alternadamente y esta prohibido pasar su turno. 
Si uno de los jugadores pudiese pasar su turno, el juego podría no terminar jamás. En efecto, si un jugador descubre que no puede ganar, él pasara su turno y el otro jugador no querrá continuar.

5. Ambos jugadores conocen perfectamente el estado del juego, es decir, cada jugador conoce la posición y el conjunto de opciones de esa posición en cada jugada.

6. El azar no interviene en las jugadas.

7. En la convención normal de un juego combinatorio, el primer jugador que no pueda jugar es el perdedor, es decir, el jugador que hace la última jugada valida, gana.

Cuando la convención inversa es adoptada, se habla de convención misère, es decir, el jugador que hace la última jugada valida pierde, como por ejemplo, el juego de Marienbad.

8. La regla del juego debe ser construida de tal manera que el juego tenga un fin en un número finito de jugadas: es decir, cada jugada debe aproximarnos al fin del juego. La partida se termina cuando uno de los jugadores ya no puede jugar.

Es decir, cada jugada debe aproximar a los jugadores al fin del juego. La partida se terminará cuando uno de ellos no pueda seguir jugando.

Entre los juegos combinatorios se encuentran, el juego de Nim, el Chomp, el Cram, el Sprouts etc. Por otro lado, entre los juegos no combinatorios están el juego del domino (por no cumplir los axiomas 4, 5, 6), el ajedrez (por los axiomas 3 y 7), los juegos de cartas (por los axiomas 1, 5 y 6), entre otros (DUCHENE, 2006).

\subsubsection{Estrategia ganadora, posición ganadora y posición perdedora en un juego combinatorio}

El objetivo en un juego combinatorio es, como en todo juego, ganar. Para esto tenemos dos alternativas, la primera es jugar al azar y dejar que el destino decida por nosotros y la segunda es encontrar un método que nos permita ganar siempre, sea cual sea el juego de nuestro adversario, esta segunda alternativa es lo que se conoce como estrategia ganadora. Definimos, entonces, una estrategia ganadora como el método/ receta/ técnica, que nos permite hacer las jugadas que nos llevarán a la victoria.

Toda posición que conduzca a la victoria es llamada posición ganadora, es decir, estamos en posición ganadora si potencialmente jugando bien (aplicando una estrategia) estamos seguros de ganar. Por el contrario, toda posición no ganadora, es perdedora, es decir una posición perdedora es una posición en la cual no estamos seguros de ganar.

Los problemas matemáticos de juegos combinatorios tienen una naturaleza heurística 
bastante compleja. En efecto, resolver un juego combinatorio corresponde ya sea, encontrar una estrategia ganadora y/o caracterizar las posiciones ganadoras y perdedoras del juego, por lo tanto las nociones recién abordadas son esenciales a la hora de resolver este tipo de juego.

Con el fin de alcanzar los objetivos de aprendizaje esperados (los saber-hacer propios de la actividad matemática), y lograr una trasposición didáctica del objeto juego combinatorio es que necesitamos un modelo adecuado, por esta razón nuestra investigación está basada en el modelo Situation de Recherche pour la Classe descrita a continuación.

\subsection{El modelo Situations de recherche pour la Classe}

La base teórica en la cual se enmarca nuestra investigación es el modelo francés Situations de recherche pour la Classe (SiRC) (GRENIER; PAYAN, 1998; GODOT, 2005; GIROUD, 2011; COLIPAN, 2014). Las SiRC son situaciones didácticas particulares que pueden ser consideradas como la transposición al aula de la actividad de un investigador en matemática. El modelo está caracterizado de la siguiente manera:

1. El problema abordado se inscribe en una problemática de investigación profesional, puede tener una, varias o ninguna solución o estar aún abierto en la matemática actual.

2. El problema inicial es de fácil acceso, no está formalizado en términos matemáticos. Es la situación que lleva al alumno al interior de las matemáticas.

3. Los métodos de resolución no están señalados, el problema se puede abordar a través de variadas pistas, es decir, se tiene la posibilidad de utilizar múltiples estrategias de resolución.

4. Los conocimientos escolares elementales son suficientes para entender y atacar el problema. El dominio conceptual en el cual se encuentra el problema, aunque no sea muy familiar, es de fácil acceso para que se pueda tomar fácilmente posesión de la situación.

5. Un problema resuelto reenvía a nuevos problemas.

6. Las variables del problema están a disposición del alumno y no del profesor (como en el caso de las variables didácticas de Bousseau). En una SiRC estas variables son llamadas variables de investigación.

El objetivo de las SiRC es producir respuestas a interrogantes. Los conceptos matemáticos que están en juego no están programados y no son restrictivos a priori, ellos están al servicio del problema y su solución. Los aprendizajes prioritarios son todos aquellos que caracterizan la actividad matemática: la experimentación, el estudio de casos particulares, 
el enunciado y estudio de conjeturas, la construcción de ejemplos y contra-ejemplos, la modelización, la elaboración y escritura de pruebas, la definición de objetos etc.

Un problema de tipo SiRC es claramente no usual en la clase. La autonomía en la investigación, implica que los alumnos puedan tratar de resolver sus propias preguntas. No hay, necesariamente, una respuesta final al problema inicial, el objetivo no es la ejercitación de un técnica ni un método visto durante una clase y es la prueba o demostración el único medio de validación a cargo de los alumnos.

\subsubsection{Un contrato didáctico especifico}

Durante la gestión de una SiRC (GRENIER; PAYAN, 2002), tanto el alumno como el profesor se encuentran en una situación diferente a la de una clase habitual. El alumno está en posición de investigador y su tarea es resolver el problema y la producción de resultados. Por otro lado, el profesor se encuentra en una doble posición: como investigador y como gestor de la situación. Como investigador porque los alumnos pueden plantearse problemas para los cuales el profesor no tiene una respuesta inmediata, de esta forma, él puede asociarse a ellos en la búsqueda de soluciones. Como gestor de la situación porque él debe controlar la actividad del alumno en relación a los objetivos de aprendizaje los saber-hacer fundamentales de la actividad matemática.

En la siguiente sección, mostramos el estudio del medio, la posición de los actores y la gestión de una SiRC en un caso particular de problema combinatorio: El juego del Chocolate.

\subsection{Metodología}

Este trabajo está basado en una metodología de investigación con un enfoque de tipo cualitativa basada en una ingeniería didáctica (ARTIGUE, 1989; ARTIGUE et al., 1995). La ingeniería didáctica es una metodología de investigación de origen francés cuya característica principal es su esquema experimental basado en la concepción, realización y análisis de secuencias de enseñanza. El registro está basado en el estudio de casos y la validación es esencialmente interna, fundada en la confrontación entre el análisis a priori y a posteriori (GODINO et al., 2013).

El proceso o desarrollo de una ingeniería didáctica está basado en cuatro fases: la fase 1 de análisis preliminar, la fase 2 de análisis a priori de la(s) situación(es) didáctica(s) de la ingeniería, la fase 3 de experimentación y, finalmente, la fase 4 de análisis a posteriori y 
validación.

En este trabajo, el análisis preliminar contempló un estudio epistemológico y didáctico de los juegos combinatorios, una investigación sobre trabajos recientes en el área y un análisis sobre su implicación en la enseñanza actual, estos análisis se encuentran de forma detallada en Colipan (2014).

El análisis a priori que corresponde al análisis descriptivo y predictivo de la situación a-didáctica, fue hecho en base al estudio del juego combinatorio El juego del Chocolate (COLIPAN, 2014), del cual se realizó un análisis matemático, que contemplo un estudio de las estrategias de investigación y análisis de casos particulares y generales además de una análisis didáctico, en el que se estudiaron las nociones, variables y conocimientos en juego en la situación. Algunos de estos elementos son presentados en la siguiente sección.

La experimentación realizada tuvo como objetivo el trabajo sobre las nociones trasversales abordadas por los alumnos y sus consecuencias en relación a la puesta en marcha de estrategias ganadoras para el juego, precisamente se trató de analizar:

- Las estrategias que pueden ser desarrolladas por los alumnos.

- Los resultados (conjeturas, contra-ejemplos, métodos, pruebas) que los alumnos pueden encontrar.

Esta, fue realizada a alumnos de primer año de universidad, de la carrera Pedagogía en Matemática, que jamás habían sido confrontados a situaciones de este tipo.

Para el análisis a posteriori y validación, las experimentaciones fueron analizadas por la vía de la dialéctica de la acción, la formulación y la validación (BROUSSEAU, 2004), utilizando la correspondencia hecha por Godot (2005) en donde:

1. La acción: Corresponde a las experimentaciones aleatorias e inductivas, la modelización y las conjeturas elaboradas.

2. Formulación: Corresponde a la modelización, conjeturas y pruebas locales.

3. Validación: Corresponde a las pruebas de validación de conjeturas globales.

A continuación presentamos parte de los análisis a priori, experimentación y análisis a posteriori realizados en torno a El juego del chocolate.

\section{El juego del chocolate}

El juego del Chocolate es un juego combinatorio, perteneciente a la batería de 
situaciones del equipo de investigación francés Maths à Modeler ${ }^{1}$, y ha sido experimentada en múltiples ocasiones, sin embargo el análisis matemático y didáctico del juego, no había sido realizado.

Regla del juego: Inicialmente se dispone de una barra rectangular de chocolate. Cada jugador debe, en su turno, quebrar la barra en dos siguiendo una línea horizontal o vertical y comer una de las dos partes. Sin embargo, uno de los cuadrados del chocolate es de jabón. El ganador es el jugador que evita comer el cuadrado de jabón.

Con el fin de poder describir las posiciones del juego en relación a los análisis que presentamos más adelante, toda posición del juego será denotada por $P=(a, b, s, t)$ con $a, b, s, t$ enteros naturales tales que $s \leq a$ y $t \leq b$, correspondiendo estos a una barra de chocolate de dimensiones $a \times b$ en donde el cuadrado de jabón está situado en la posición $(s, t)$.

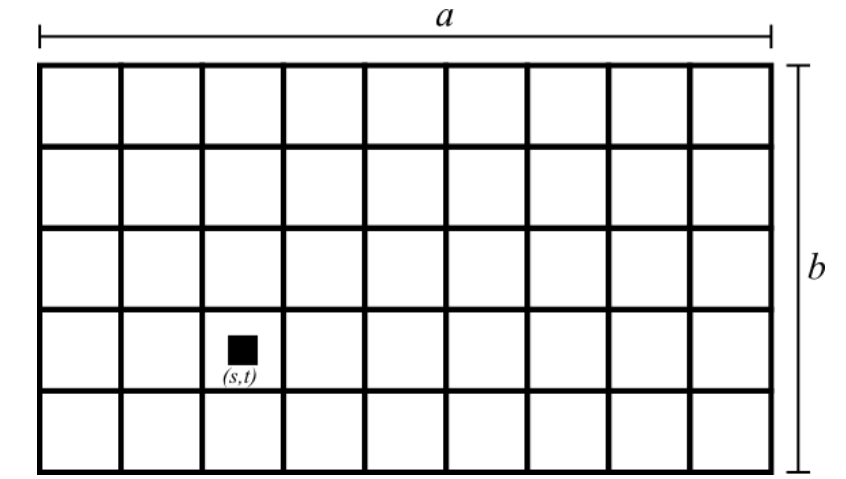

Figura 9 - Barra de chocolate de posición $P=(a, b, s, t)$.

Ejemplo: Los turnos sucesivos de una partida del juego del chocolate en una barra de dimensiones $4 \times 2$ con el cuadrado con jabón situado en $(s, t)=(2,2)$ son:
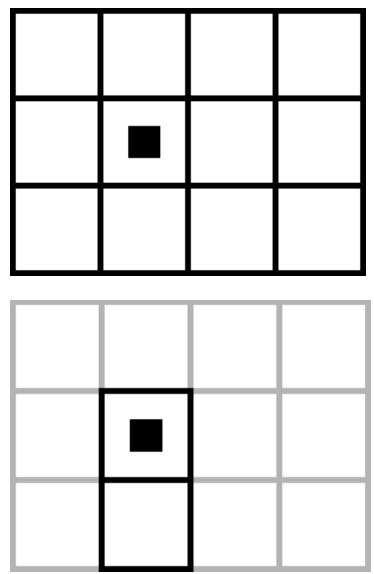
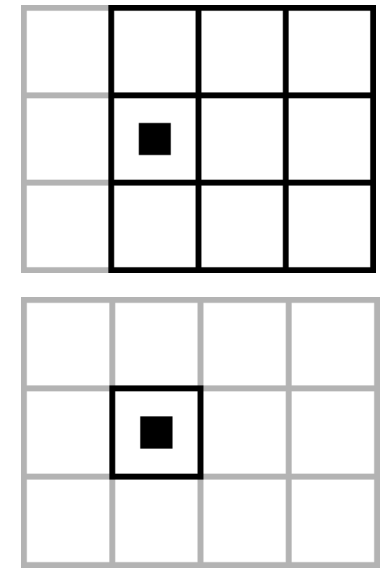

Figura 10 - Ejemplo del juego chocolate.
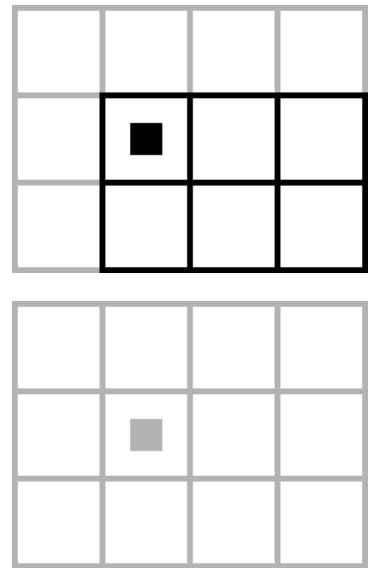

El color gris claro, denota la porción de chocolate que ha sido tomada. La partida

\footnotetext{
${ }^{1}$ La autora es miembro permanente del equipo Maths à Modeler, desde el año 2009, este trabajo se enmarca en una tesis doctoral realizada en el seno del equipo.
} 
consta de 5 jugadas (la primera figura corresponde a la posición de inicio), y es el primer jugador el que toma el cuadrado con jabón, por lo tanto, pierde la partida.

\subsection{Análisis a priori de el juego del chocolate}

En una primera instancia, la resolución de la situación El juego del Chocolate, se puede desarrollar en dos etapas:

- La búsqueda de casos particulares fácilmente abordables.

- La validación de las conjeturas encontradas en el estudio de estos casos particulares.

$\mathrm{Al}$ resolver el juego, podemos observar que las dimensiones de la barra de chocolate y posición del cuadrado con jabón constituyen lo que se llama variable de investigación en una SiRC. En efecto, si el problema es propuesto de manera abierta, (como se presentó en la regla del juego) los valores de estas variables quedan a disposición del alumno y según el valor que estas tomen, el avance en el problema será diferente, por lo tanto, para comenzar a encontrar pistas de solución y avanzar en la resolución, podemos, por ejemplo:

- Fijar $(s, t)$, es decir, la posición de cuadrado con jabón. Por ejemplo fijarlo en una esquina.

- Fijar $(a, b)$, es decir, las dimensiones de la barra de Chocolate. Por ejemplo trabajar con un chocolate de dimensión $a \times 1$.

- Fijar $(a, b, s, t)$ Por ejemplo, fijar el valor del jabón en una esquina y trabajar con barras cuyas dimensiones son, por ejemplo, $a \times a, a \times 2$, etc.

La descripción de posiciones ganadoras y perdedoras y una estrategia ganadora del juego del chocolate pueden ser consultadas en Colipan (2014). En este artículo, mostramos algunos estudios de casos particulares y damos algunas pistas de investigación con el objetivo de motivar al lector a la búsqueda de estrategias ganadoras para este juego.

\subsubsection{Nociones en juego en la situación}

Si bien en una SiRC las nociones en juego no son objetivo de aprendizaje, cada situación pone en juego nociones que aparecen en el proceso de resolución, de esta manera, en el juego del chocolate a nivel de nociones podemos encontrar:

1. Nociones matemáticas: Estas pueden aparecer en función del método elegido para 
abordar el problema, a saber, desarrollar y aplicar conceptos de la teoría de números ligadas a números primos, factores, múltiplos y división euclidiana; usos de las clases de equivalencia en los enteros modulo $p$, usos de la simetría, entre otros.

2. Nociones específicas de un juego combinatorio: Como hemos visto en la sección 2.2.2, los juegos combinatorios pone en juego nociones especificas tales como: estrategia, estrategia ganadora, posición perdedora, posición ganadora.

3. Saber-hacer fundamentales de la matemática: El juego del chocolate pone en obra saber-hacer propios de la actividad matemática, específicamente, los elementos necesarios para formular y validar resultados concernientes al juego, estos elementos se desarrollan con más profundidad en las siguientes subsecciones.

\subsubsection{Formulación de conjeturas}

\section{a) Experimentaciones aleatorias}

Durante un primer tiempo, es muy probable que exista una fase de experimentación aleatoria en la cual, los alumnos, jugarán en barras de chocolate elegidas al azar. El que gestiona la situación, podrá proponer posteriormente a los alumnos, comenzar a estudiar casos particulares. Estas experimentaciones van a ayudar al alumno a apropiarse del juego y a descubrir características como por ejemplo:

- Las posiciones perdedoras sobre un chocolate de dimensión $a \times 1$ y en barras de chocolate con dimensiones pequeñas, con el cuadrado de jabón en una esquina, son todas simétricas.

- Los casos particulares de dimensiones $a \times 1$ y $a \times a$ con el cuadrado de jabón situado en una esquina pueden ser probadas usando argumentos de simetría. En efecto, una posición simétrica es perdedora porque el adversario puede imitar mis jugadas hasta que quede solo el cuadrado de jabón. Toda otra posición es ganadora, porque posee una posición simétrica como opción de jugada.

- El caso particular de dimensiones $a \times 2$ es un poco más difícil de analizar, pero esta puede ser analizada con ayuda de la resolución del caso $a \times 1$.

b) Experimentaciones inductivas

Después de las experimentaciones aleatorias para la apropiación del juego, tenemos la hipótesis que los alumnos pueden pasar a una fase de experimentación inductiva. En esta fase los alumnos pueden estudiar barras de chocolates especialmente elegidas por ellos, para 
elaborar conjeturas sobre su estrategia ganadora. La naturaleza de las experimentaciones en esta etapa dependerá fuertemente de las propiedades identificadas durante la fase aleatoria, como por ejemplo:

- Los alumnos que remarcan que las posiciones simétricas de tallas pequeñas son perdedoras pueden analizar las posiciones más grandes para después hacer conjeturas en relación a la simetría de posiciones.

- Los alumnos que remarcan directamente que las posiciones simétricas son perdedoras pueden estudiar las posiciones que tienen una opción simétrica para enunciar una conjetura.

\subsubsection{Validación de conjeturas}

Los argumentos para validar conjeturas pueden ser de diferentes tipos.

a) Experimentaciones repetitivas y validativas.

Una experimentación es llamada repetitiva cuando, después de algunas experiencias aleatorias, la conjetura se confirma. Tal como afirma Giroud (2011) no es absurdo pensar que si un resultado se repite reiteradamente entonces es verdadero, sin embargo, en matemática esto es insuficiente.

Una experimentación es validativa si ella tiene como objetivo la búsqueda de un contra-ejemplo para una conjetura en particular, si no es posible encontrar un contra-ejemplo entonces la conjetura es considerada valida, sin embargo este tipo de razonamiento puede llevar a una prueba por exhaustividad de casos si restringimos el juego para tener un número finito de casos. Por ejemplo la posición de partida $P=(6,2,3,1)$ es bastante interesante en el hecho que aporta información sobre el juego, además puede ser analizada por exhaustividad de caso, ya que ella solo tiene 6 opciones.

\section{b) Argumentos matemáticos}

Los argumentos matemáticos aparecen cuando se describen las posibles pistas de investigación y una estrategia ganadora para el juego. Estos argumentos fueron presentados en Colipan (2014) con un lenguaje matemático que puede estar fuera del alcance de un alumno de secundaria o de primeros años de universidad, sin embargo el contenido de los argumentos de demostración pueden ser utilizados por los alumnos en forma menos elaborada.

\section{c) Ejemplo genérico}

En el caso particular de una barra de chocolate con el cuadrado de jabón, en una esquina, un ejemplo genérico puede ser cualquier rectángulo bastante grande para que su 
análisis permita identificar que las posiciones simétricas son las posiciones perdedoras, por ejemplo la posición inicial $P=(6,7,1,1)$. Lo mismo ocurre para el juego en una barra de chocolate de dimensión $a \times 1$, un ejemplo genérico que permite reconocer la naturaleza simétrica de las posiciones perdedoras es $P=(12,1,6,1)$.

Sin embargo, para el caso general (cualquier dimensión del chocolate, y el cuadrado de jabón en una posición arbitraria) creemos que ningún ejemplo es capaz de tener toda la información de la situación.

\subsubsection{Elementos de validación}

\section{a) Prueba por exhaustividad de casos}

Podemos realizar pruebas por exhaustividad de casos, como dijimos anteriormente, cuando las dimensiones de la barra de chocolate son pequeñas.

\section{b) Contraejemplos}

Un contraejemplo bien elegido es suficiente para invalidar una conjetura, el problema radica en que puede ser difícil encontrarlo, en este caso, el gestor de la situación puede ayudar a la construcción de estos contra-ejemplos y ayudar a los alumnos a progresar en el análisis del juego sin dar pistas de la solución.

c) Validación de una estrategia ganadora

Una vez que los alumnos hayan encontrado una conjetura (una estrategia ganadora) para el caso general o para una familia infinita de casos, surge el problema de como validar esta conjetura. Considerando que una infinidad de familias hace imposible una prueba por exhaustividad de casos, los alumnos deberán encontrar los métodos para hacer pruebas generales.

Es difícil demostrar que una estrategia cualquiera es, efectivamente, una estrategia ganadora. La manera más rigurosa de hacerlo es introducir la noción de posición ganadora y posición perdedora y mostrar que la estrategia en cuestión, aplicada a una posición ganadora, da una posición perdedora.

\subsubsection{Variables de la situación}

a) Variable didáctica ligada al soporte físico

Podemos elegir diferentes tipos de soporte físico: lápiz-papel, en línea a través de un 
computador $^{2}$, con material manipulable como por ejemplo, chocolates de cartón que se puedan ir cortando etc.

b) Variable de investigación ligada al valor numérico de $(a, b)$ y $(s, t)$

A partir de los valores de $(a, b)$ y $(s, t)$ podemos obtener los datos necesarios para jugar y resolver el juego del chocolate. Las diferentes valores de $P=(a, b, s, t)$ conducen a conjeturas diferentes, propias a la identificación de situaciones ganadoras y perdedoras para cada sub-problema.

c) Variable de investigación ligada a la cantidad de cuadrados de chocolate a tomar en cada turno

Si bien en el enunciado del juego no se han introducido restricciones para el número máximo de filas o columnas a extraer en cada turno, se podría también analizar una versión de sustracción del juego del chocolate en el cual solamente un numero establecido de filas o columnas se pueden extraer en cada turno, esta generalización del juego es un problema aún abierto en matemática.

\section{Producción de los alumnos}

El total de alumnos que participaron en la experiencia fue de 50 estudiantes, durante un total de 4 horas, repartidas en 2 sesiones. Las conversaciones de 5 grupos fueron registradas a través de grabadoras vocales y filmadoras, pero con el fin de recolectar la mayor cantidad posible de información, cuatro observadores tomaban notas entre los grupos.

Los participantes fueron organizados en grupos de 3 o 4, cada grupo dispuso del enunciado, de hojas blancas y lápices de diferentes colores para identificar todas las jugadas. La gestión de la experimentación estuvo a cargo de dos investigadores. Ellos circulaban entre los grupos con el fin de verificar que el problema había sido entendido y ayudar a los estudiantes en sus investigaciones. Sin embargo, sus repuestas eran neutras, sin ofrecer ninguna pista del resultado. A continuación, presentamos el análisis de las experiencias realizadas.

\subsection{Devolución de la situación}

La devolución fue rápida y activa en todos los grupos. Una vez entregadas las reglas

\footnotetext{
${ }^{2}$ En el sitio del equipo maths à modeler puede encontrar varias SiRC, entre ellas el juego del chocolate, en el cual es posible jugar contra el computador. http://mathsamodeler.ujf-grenoble.fr/LAVALISE/debutval.php
} 
del juego, además de un ejemplo, todos los grupos se sumergieron en la búsqueda de estrategias ganadoras. Por lo tanto, la devolución de la actividad de investigación se realizó de manera autónoma y sin dificultad.

\subsection{Estrategia ganadora, posición ganadora, posición perdedora.}

Estas nociones fueron bien asimiladas y operacionales, por los alumnos, incluso de manera implícita, en el estudio de métodos de investigación y elaboración de conjeturas. En torno a la noción de estrategia ganadora se identificaron los siguientes elementos en torno al juego:

- La diferencia entre una victoria debida al azar y una victoria debida a una estrategia ganadora.

- Siempre existe un ganador y un perdedor.

- La estrategia ganadora depende solo de la posición del instante del juego.

En el caso de las nociones de posición ganadora y perdedora, los alumnos identifican bien la situación de superioridad que implica una posición ganadora, sin embargo la noción de posición perdedora fue un poco más difícil de asimilar en el sentido que encontrarse en una posición perdedora no significa forzosamente que se va a perder de manera absoluta.

"según lo que quede de chocolate, yo tengo la posibilidad de hacer una jugada que nos llevará hacia la victoria (estoy en posición ganadora) o no (estoy en posición perdedora)" (Grupo A).

La figura muestra las conclusiones de un grupo, en relación al jugador que se encuentra en posición ganadora para diez casos particulares de posiciones de juego.

\begin{tabular}{|c|c|c|}
\hline jugades & jugados 1 & jugador 2 \\
\hline$I$ & & $\checkmark$ \\
\hline II & & $\checkmark$ \\
\hline III & & $V$ \\
\hline IV & & $\checkmark$ \\
\hline$\underline{E}$ & $\checkmark$ & \\
\hline$\overline{\text { VI }}$ & & $\checkmark$ \\
\hline$\underline{\underline{\text { VII }}}$ & $\checkmark$ & \\
\hline VIII & & $\checkmark$ \\
\hline$\underline{I x}$ & $\checkmark$ & \\
\hline$I$ & $V$ & \\
\hline
\end{tabular}

Figura 11 - Estudio posición ganadora y posición perdedora.

La siguiente figura, muestra los resultados de un grupo con respecto a una estrategia ganadora para el caso de un chocolate de dimensión $a \times 1$. 

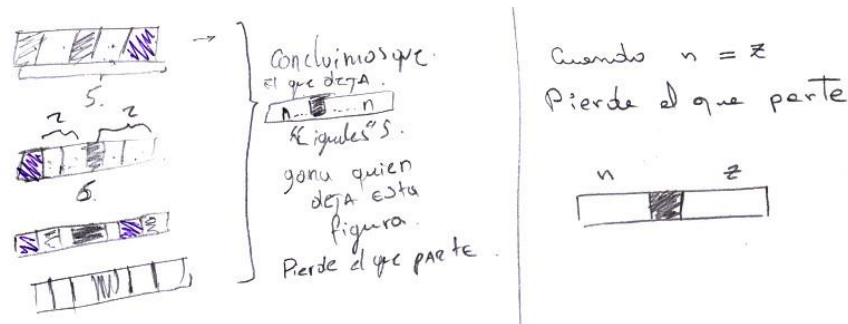

Figura 12 - Estrategia ganadora para un chocolate de dimensión $a \times 1$.

\subsection{Métodos de investigación y conjeturas}

Todos los grupos fueron capaces de enunciar conjeturas, por supuesto que no todas han sido correctas, los elementos de validación e invalidación han sido ejemplos y contraejemplos. Durante las sesiones, la única prueba o demostración realizada, fue la exhaustividad de casos.

Por otro lado, soluciones particulares han sido exhibidas por casi todos los grupos. La búsqueda de estrategias ganadoras ya sean locales o globales, no fue igual en los todos grupos. Algunos se quedaron a nivel local, otros progresaron hacia la generalización. A continuación un resumen de las conjeturas y estrategias locales y globales encontradas.

a) Estudio del chocolate de dimensiones $a \times 1$ :

- Si una de las posiciones es simétrica, con respecto al cuadrado de jabón, entonces ella es perdedora. La estrategia ganadora es para el segundo jugador que tiene como opción imitar las jugadas del primero.

"Para $a$ impar y jabón en el centro. El jugador 2 puede siempre hacer la misma jugada que el primero. El segundo gana" (grupo A).

- Si a es un número par, la posición es ganadora con la estrategia de hacer una jugada que de una posición simétrica a nuestro adversario.

"Para a par: siempre habrá más chocolate a un lado del jabón que del otro. El primero tiene la opción de igualar la cantidad de chocolate y así hacer jugadas idénticas al segundo jugador" (grupo C).

- Una posición es perdedora si y solamente si ella es simétrica.

"El segundo siempre gana a menos que el número de filas a cada uno de los lados del jabón sea igual” (grupo A).

- Una posición es perdedora si y solamente si ella es simétrica. Si la posición no es simétrica, la jugada ganadora es jugar la única opción simétrica.

"El caso de que la barra sea una columna partido por $n$ filas siempre gana el 
primero igualando el número de filas a cada uno de los lados del jabón, a menos que ya estén igualados (y siempre y cuando el jabón no esté en los extremos)" (grupo B).

b) Estudio del chocolate de dimensiones a $\times 2$ : conjeturas.

- La posición $(2,2,1,1)$ es perdedora.

"En un chocolate de $2 \times 2$, por simetría, gana el segundo" (grupo D).

- Reducción a la dimensión $a \times 1$ :

"Hay que tratar de dejar el chocolate a una columna (siempre y cuando el jabón no esté en una esquina)" (grupo B).

"No importa la cantidad de cuadrados que hayan, siempre hay que llegar al caso a $\times 1 "$ (grupo A).

- Las posiciones $(2 s-1,2, s, 1)$ y $(a, 2,1,1)$ son ganadoras.

"Gana si se deja al segundo jugador una figura de la forma: $a \times 1$ con el jabón al medio y a $\times 1$ con el jabón en la esquina" (grupo C).

c) Estudio del chocolate con el cuadrado con jabón en una esquina.

- Si la forma del chocolate no es cuadrada, la posición es ganadora. La jugada ganadora es volverla cuadrada.

"En el caso de que el chocolate sea un rectángulo y el jabón esté en uno de los extremos siempre gana el primero si es que logra hacer un cuadrado en el que esté incluido el jabón” (grupo A).

"En cuadrilátero de $\mathrm{a} \times \mathrm{b}$, el jugador debe forzar un cuadrado para ganar, cada vez que el contrincante desarme el cuadrado el jugador debe volver a formarlo, hasta llegar a un cuadrilátero de $2 \times 2$, obligando al contricante a perder" (grupo B).

- Si el chocolate no es cuadrado, la posición es ganadora.

"Cuando se dé el caso en que los lados son desiguales gana el segundo" (grupo D).

- La posición $(2,2,1,1)$ es perdedora.

"Si el jabón está en una esquina, gana el que trata de dejar un cuadrado de $2 \times 2$ " (grupo A).

- Las opciones $(a, 1,1,1)$ son siempre ganadoras $(a \geq 2)$.

"Pierde el jugador que deja una columna y el jabón en una esquina” (grupo D). La elaboración y estudio de estas estrategias nos muestra que los alumnos son capaces 
de formular conjeturas y estudiarlas. De esta manera una vez que la conjetura es precisada, el método de los alumnos consistió, ya sea, en seguir la experimentación para mejorar la conjetura o en considerar que ella es verdadera y pasar a la resolución de otro problema. En este sentido hemos constatado que las intervenciones del gestor fueron las que incitaron a los alumnos a construir demostraciones, como veremos a continuación la noción de contraejemplo jugo un rol particularmente importante a nivel de validación.

\subsection{Elementos de validación}

En todos los casos, la validación de conjeturas fueron hechas de manera casi formal, para los casos pequeños, la mayoría se apoyaban sobre la exhaustividad de casos. Para el caso del chocolate con dimensiones $a \times a$ y $a \times 1$ las pruebas realizadas eran fundamentadas en la simetría y la estrategia de imitar.

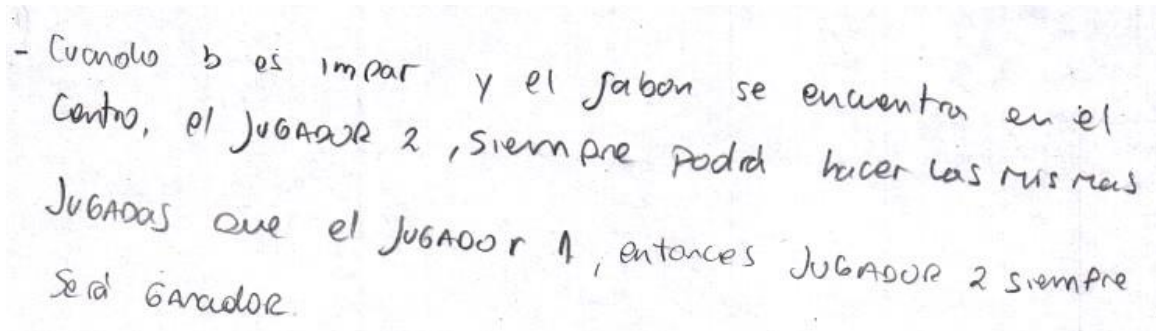

Figura 13 - Estrategia utilizando simetría.

Por otro lado, las conjeturas falsas enunciadas durante las sesiones fueron, la mayoría de ellas, invalidadas a través de contra-ejemplos por los propios grupos.

De esta forma, a pesar de la complexidad del juego, los contra-ejemplos fueron pertinentes, fáciles de comprender, accesibles y convencedores.

\subsection{La expresión escrita y oral}

Tanto la expresión escrita como la oral fue un freno para la re trascripción de ideas. En efecto, en la mayoría de los grupos los alumnos mostraban serios problemas para encontrar las palabras que describían sus acciones, creemos que esto puede ser debido a la ausencia de esta práctica en la enseñanza.

\subsection{Desarrollo de la gestión de la situación}

Introducir este tipo de actividades en clase implica la puesta en marcha de condiciones 
específicas de gestión. La persona encargada de la gestión de la situación es indispensable ya que este juega un rol determinante, basado en los saber-hacer específicos tales como: introducir una nueva problemática en el momento justo, ayudar a los alumnos en la formulación de sus respuestas sin validarlas, o al contrario validarlas o invalidarlas, por ejemplo, a través de contra-ejemplos e incitar a los alumnos a justificar sus resultados.

\section{Conclusión}

La situación El Juego del Chocolate pone en obra saber-hacer fundamentales de la actividad matemática tales como: la experimentación sobre pruebas aleatorias (jugando al azar), la experimentación sobre casos particulares, la construcción de conjeturas (en términos de estrategias ganadoras), la invalidación de conjeturas (a través de contra-ejemplos) o validación (a través de la exhaustividad de casos o ejemplos). De esta manera, podemos decir que las situaciones basadas en juegos combinatorios, inducen una actividad matemática que va más allá del desarrollo y la práctica de técnicas propias de las matemática, ellas abren el acceso a saber-hacer más generales, que pueden ser fuente de aprendizaje de los saber-hacer propios de la actividad matemática.

Por otro lado, la percepción que tenga el gestor sobre la situación y su rol serán fundamentales. El gestor debe estar convencido que en una situación de juego es posible crear el medio para una situación a-didáctica en matemática, que el objetivo fundamental es implicar a los alumnos en la resolución de un problema y que la búsqueda de soluciones por parte de los alumnos es más interesante que el resultado mismo. Para esto es necesario, a la vez, aceptar los aportes intuitivos y personales.

Finalmente, podemos decir que una situación didáctica creada entorno a los juegos combinatorios puede generar aprendizajes significativos en los alumnos en la medida que los objetivos vayan más allá de la contingencia del programa escolar. La finalidad de estas situaciones debe ser la intervención de manera autónoma y efectiva del alumno, sin por ello limitarlos a aprender una noción o procedimiento en particular.

\section{Reconocimiento}

Trabajo realizado en el marco del proyecto de investigación, 72090110. De la Comisión Nacional de Ciencia y Tecnología (CONICYT), Chile. 


\section{Referencias}

ARTIGUE, M. Ingénierie didactique. Recherches en Didactique des Mathématiques, Grenoble, v. 9 (3), p. 281-308, 1989.

ARTIGUE, M; DOUADY, R; MORENO, L.; GOMEZ, P. Ingeniería Didáctica en Educación

Matemática, Un esquema para la investigación y la innovación en la enseñanza y el aprendizaje de las Matemáticas. Bogotá: Grupo Editorial Iberoamérica, 1995.

BERLEKAMP, E.; CONWAY, J.; GUY, R. Winning ways for your mathematical plays. Natick, Wellesley: A. K. Peters Ltd, 2001.

BOUTON, C. Nim, a game with a complete mathematical theory. The Annals of Mathematics, Cambridge, v. 3, p. 35-39, 1901.

BROUSSEAU, G. Théorie des situations didactiques. Grenoble : La pensée sauvage éditions, 2004.

CAMPANARIO, J.; MOYA, A. ¿Cómo enseñar ciencias? Principales tendencias y propuestas. Enseñanza de las ciencias, Barcelona, v. 17, p. 179-192, 1999.

COLIPAN, X. Étude didactique des situations recherche pour la classe concernant des jeux combinatoires de type Nim, 2014. 384f. Thèse (Doctorat en didactique de mathématique) - Institut Joseph Fourier, Université de Grenoble 1, Grenoble, 2014.

COLIPAN, X.; GRENIER, D. Le jeu d'Euclide géométrique, une situation recherche pour construire et développer les savoir-faire fondamentaux de l'activité mathématique. Submitted to PETIT X. Grenoble. 2016.

COPPÉ, S.; HOUDEMENT, C. Réflexions sur les activités concernant la résolution de problèmes à l'école primaire. Grand N, Grenoble, v. 69, p. 53-63, 2002.

DUCHENE, E. Jeux combinatoires sur les graphes, 2006. 157f. Thèse (Doctorat en mathématique) - Institut Joseph Fourier, Université Joseph Fourier, Grenoble, 2006.

HALL, O. La Desafección de los Jóvenes por la Ciencia y la Tecnología: Una Tendencia Peligrosa. Paris: Documento Unesco, 2012.

GIROUD, N. Étude de la démarche expérimentale dans les situations de recherche pour la classe, 2011. 610f. Thèse (Doctorat en didactique de mathématique) - Institut Joseph Fourier, Université de Grenoble 1, Grenoble, 2011.

GODINO, J.; BATANERO, C.; CONTRERAS, A.; ESTEPA, A.; LACASTA, E.; WILHELMI, M. Didactic engineering as design-based research in mathematics education. In: CONGRESS OF EUROPEAN RESEARCH IN MATHEMATICS EDUCATION, CERME, 8. 2013. Ankara, Turquía. Proceedings... Ankara: Middle East Technical University, 2013. p. 2810-2819.

GODOT, K. Situations recherche et jeux mathématiques pour la formation et la vulgarisation, exemple de la rue aux couleurs, 2005. 407f. Thèse (Doctorat en mathématique) - Institut Joseph Fourier, Université Joseph Fourier, Grenoble, 2005.

GRENIER, D ; PAYAN, C. Spécificités de la preuve et de la modélisation en Mathématiques Discrètes. Recherches en didactique des mathématiques, Grenoble, v. 18 (2), p. 59-100, 1998.

GRENIER, D. ; PAYAN, C. Situations de recherche en classe: essai de caractérisation et proposition de modélisation., 2002. Paris. In: Séminaire national de didactique de mathématiques, 7. Paris, 2002. Actas... Paris : IREM de Paris 7 et ARDM, 2002. p. 189-205. 
GRAVIER, S.; OUVRIER-BUFFET, C. Maths à Modeler: Research-Situations for Teaching Mathematics. In: ICMI STUDY, 16. 2009, Toronto. Proceedings...Barbeau, Toronto: E. \& Taylor, 2009. p. 23-29.

OUVRIER-BUFFET, C. ; PERRIN, M. Apprendre à faire des mathématiques du primaire au supérieur-Quoi de neuf? Approches plurielles en didactique des mathématiques. Paris: Université Paris Diderot - Paris 7, 2009.

TISSERON, C. Développer la recherche scientifique à travers l'étude de situations mathématiques. Université d'été. 1996, Lyon. Actes de l'université d'été. Lyon: Ed. Magnard. p. 29-39, 1996.

Submetido em Abril de 2015. Aprovado em Outubro de 2015. 